\title{
Produção científica no Brasil sobre álcool e mulher: uma revisão bibliográfica
}

\author{
Scientific Production on Alcohol and Women in Brazil: a Literature Review \\ Izete Soares da Silva Dantas Pereira*
}

\begin{abstract}
Resumo:
Comportamento conhecido como predominantemente masculino, o uso/abuso de álcool vem ganhando protagonismo também nas mulheres, com aumento significativo nos últimos anos. Realizou-se uma pesquisa bibliográfica para o levantamento da literatura científica brasileira que faz referência ao uso de álcool pelas mulheres, nomeadamente aquela produzida na forma de artigos publicados. A coleta de dados ocorreu nos meses de novembro de 2009 a julho de 2010, com base no Banco de Dados da interface Scielo, correspondente ao período de 2000 a 2009, totalizando dez anos de publicações. Foram definidas seis categorias de análise, encontrando-se a maior percentagem de publicações nas categorias "Uso de álcool e comportamentos de risco", seguida de "Álcool e violência". Conclui-se pela necessidade de incrementar os estudos sobre a temática, incluindo o gênero feminino como uma importante variável a ser considerada nas políticas públicas de enfrentamento e combate ao alcoolismo, bem como nas estratégias de prevenção e/ou tratamento.
\end{abstract}

Palavras-Chave: Álcool e mulher. Pesquisa Bibliográfica. Produção Científica. Comportamentos de Riscos.

\begin{abstract}
:
Behavior known as predominantly masculine, the use/abuse of alcohol has been gaining prominence also in women, with significant increase in the last years. It was performed a survey on the brazilian scientific literature that references alcohol use by women, particularly the one produced in the form of published articles. Data collection occurred from November 2009 to July 2010, based on the database of SCIELO platform corresponding to the period of 2000 to 2009, totaling ten years of publications. There were defined six categories of analysis and the highest percentage of publications were in the categories of Alcohol use and risky behaviors, followed by Alcohol and violence. There is the need to increase studies on the subject, including the female gender as an important variable to be considered in public policies of confrontation and combat, as well in prevention and/or treatment strategies.
\end{abstract}

Keywords: Alcohol and women. Bibliographic Search. Scientific Production. Risky Behavior.

\footnotetext{
* Universidade do Estado do Rio Grande do Norte/UERN. Assistente Social, Mestre e Doutora em Saúde Pública/Universidade de São Paulo/USP.E-mail: izetedantas@hotmail.com; izetedantas@yahoo.com.br.
} 


\section{Introdução}

O álcool é a droga mais consumida em todo o mundo, segundo dados da Organização Mundial de Saúde (OMS). Fazem uso social ou problemático dessa substância cerca de 2 bilhões de pessoas. Seu uso indevido é um dos principais fatores que contribuem para a diminuição da saúde mundial, sendo responsável por 3,2\% de todas as mortes e por $4 \%$ de todos os anos perdidos de vida útil. Em relação à América Latina, o álcool assume uma importância ainda maior quando esses índices são analisados, uma vez que $16 \%$ dos anos de vida útil perdidos neste continente estão relacionados ao uso indevido dessa substância, sendo quatro vezes maior do que a média mundial (LARANJEIRA, et al., 2007).

O consumo de bebidas alcoólicas é um comportamento existente na maioria das culturas. Seu uso tem sido associado a cerimônias religiosas, eventos culturais, situações de negócio e celebrações sociais. No entanto, o consumo nocivo de álcool é responsável por cerca de $3 \%$ de todas as mortes que ocorrem no mundo, incluindo cirrose e câncer hepático até acidentes, quedas, intoxicações e homicídios Nos países em desenvolvimento, entre eles o Brasil, as bebidas alcoólicas são um dos principais fatores de doença e mortalidade, com seu impacto deletério, sendo considerado entre $8 \%$ e 14,9\% do total de problemas de saúde dessa população (MELONI; LARANJEIRA, 2004).

O consumo abusivo de álcool, ou seja, abuso/dependência estão associados a múltiplas consequências adversas para a saúde, como doenças cardíacas e cerebrovasculares, eventos fatais e transtornos psiquiátricos, traumas, violência doméstica, quedas, várias neoplasias, doenças sexualmente transmissíveis, cirrose hepática, mortes no trânsito, suicídios, desentendimentos familiares e afetivos, sendo, também, associado a homicídios, violência contra crianças e mulheres, abandono do trabalho, evasão escolar, entre outros (GUIMARÃES, et al., 2010).

O álcool é, possivelmente, a única substância de abuso que a maioria dos adultos do mundo ocidental já experimentou e uma proporção considerável o usa periodicamente durante a maior parte de sua vida. Assim, dados de comparações entre taxas de uso de álcool na vida em diferentes grupos etários, sexo e regiões ou países não são esclarecedores. 
Os problemas associados ao consumo de bebidas alcoólicas têm sido reconhecidos, de forma cada vez mais crescente, como uma questão de saúde pública em muitos países, e também no Brasil. O alcoolismo é o conjunto de problemas relacionado ao consumo excessivo e prolongado do álcool. É entendido como o vício de ingestão excessiva e regular de bebidas alcoólicas e todas as consequências decorrentes (BRASIL, 2008; NÓBREGA; OLIVEIRA, 2005).

Em 2001, foi realizado pela Secretaria Nacional Antidrogas (SENAD) em parceria com o Centro Brasileiro de Informações sobre Drogas (CEBRID) o I Levantamento Domiciliar sobre o Uso de Drogas Psicotrópicas no Brasil, um estudo envolvendo as 107 maiores cidades do país. Este estudo mostrou informações atualizadas e padronizadas sobre a prevalência do uso de álcool e outras drogas pela população. Os sujeitos da pesquisa foram pessoas com idade compreendida entre 12 e 65 anos, de ambos os sexos. Os resultados mostraram que $68,7 \%$ delas já haviam feito uso de álcool alguma vez na vida. Além disso, $11,2 \%$ da população brasileira apresentavam dependência desta substância (CARLINI et al., 2002).

Os dados do II Levantamento Domiciliar sobre o Uso de Drogas Psicotrópicas no Brasil, realizado em 2005 por pesquisadores do CEBRID (CARLINI; GALDUROZ, 2007), envolvendo as 128 maiores cidades do país, apontam que $12,3 \%$ das pessoas, com idade entre 12 e 65 anos, são dependentes do álcool, taxa superior à encontrada no I Levantamento (CARLINI et al., 2002). Os resultados gerais, por região, mostraram que na Região Nordeste o percentual encontrado foi de $13,8 \%$, com um maior número de dependentes do álcool do que a Região Sul, 9,0\%; Região Sudeste,12,7\%; Centro-Oeste, 12,7\%; e Região Norte $8,7 \%$. Nessa pesquisa constatou-se, ainda, que cerca de $75 \%$ dos entrevistados já beberam alguma vez na vida, $50 \%$ no último ano e $38 \%$ nos últimos 30 dias (CARLINI; GALDUROZ, 2007).

Um estudo realizado sobre o assunto, pela Secretaria Nacional Antidrogas em parceria com a Universidade Federal de São Paulo (UNIFESP), chamado de I Levantamento Nacional sobre os Padrões de Consumo de Álcool na População Brasileira, foi feito entre novembro de 2005 e abril de 2006, em 143 municípios do país. Foram realizadas 3.007 entrevistas, sendo 2.346 adultos com mais de 18 anos e 661 adolescentes entre 14 e 17 anos, em 143 municípios de norte a sul do país. A pesquisa 
detectou que 52\% dos brasileiros acima de 18 anos fazem uso de bebida alcoólica pelo menos uma vez ao ano. Entre esses, $65 \%$ são homens e $41 \%$, mulheres. Do total dos homens adultos participantes da pesquisa, $11 \%$ bebem todos os dias e $28 \%$ de um a quatro vezes por semana, estes são os que bebem "muito freqüentemente". Constatouse, ainda, que $48 \%$ são abstêmios, que nunca bebem ou que bebem menos de 1 vez por ano. No grupo dos adultos que bebem, $60 \%$ dos homens e $33 \%$ das mulheres consumiram 5 doses ou mais no último ano em que mais beberam.

Os dados encontrados são representativos de $100 \%$ da população brasileira, com exceção da população indígena e das populações que vivem em locais de convívio coletivo, como quartéis, asilos, internatos, entre outros (LARANJEIRA et al., 2007). O estudo mostrou que quase um terço dos bebedores pesados (beber em volume excessivo pelo menos uma vez no último ano) é do sexo feminino. Em duas décadas, a proporção de mulheres entre a população que consome bebidas alcoólicas triplicou (de 10\% para $30 \%)$. E o que é mais grave, a proximidade entre os gêneros é muito maior entre os adolescentes. Para jovens de 14 a 17 anos, 6\% das jovens e 10\% dos rapazes foram classificados como bebedores frequentes (cinco doses ou mais ingeridas no mínimo uma vez por semana). Os que bebem cinco doses ou mais de uma a três vezes por mês (ocasionais) são $16 \%$ dos rapazes e $14 \%$ das jovens. Os dados também mostraram que o consumo de álcool ocorre em faixas etárias cada vez mais precoces, sugerindo a necessidade de revisão das medidas de controle, prevenção e tratamento desse consumo (LARANJEIRA et al., 2007).

Pesquisas realizadas por diferentes autores e instituições em prontos-socorros de Brasília, Curitiba, Recife, Salvador, São Paulo e Campinas também constataram a presença de álcool no sangue de vítimas de acidentes em porcentagens que variaram de 29 a $61 \%$. Em relação a distúrbios mentais propriamente ditos, o diagnóstico de síndrome de dependência do álcool vem tendo destaque absoluto na proporção de internações psiquiátricas por drogas no Brasil, variando entre 90 e 95\% do total, desde os anos 1980 (CARLINI et al., 2002).

Outros estudos nacionais e internacionais têm demonstrado a ocorrência significativa de mortes e doenças associadas ao uso indevido de álcool. Relatos de violência doméstica, lesões corporais, tentativas e homicídios consumados, assim como 
outras situações de conflitos interpessoais são cada vez mais evidentes em contextos nos quais o álcool se faz presente (LARANJEIRA et al., 2007).

Conforme evidenciam esses estudos, dentre as drogas, o álcool é considerado a mais consumida. Nele está incluída uma grande variedade de substâncias diferentes em suas propriedades físicas e químicas, mas que, no entanto, possuem a característica comum de provocar uma diminuição da atividade de certos sistemas específicos do Sistema nervoso Central (SNC), podendo ocorrer diminuição da atividade motora, da reatividade à dor, ansiedade, euforia inicial e, posteriormente, um aumento da sonolência (BRASIL, 2008; FORNAZIER; SIQUEIRA, 2006; LARANJEIRA; DUALIBI; PINSKY, 2005).

A relação do indivíduo com uma substância psicoativa pode, dependendo do contexto, ser inofensiva ou apresentar poucos riscos, mas também pode assumir padrões de utilização disfuncionais, com prejuízos biológicos, psicológicos e sociais. Isso justifica os esforços para difundir informações básicas e confiáveis a respeito de um dos maiores problemas de saúde pública que afeta, direta ou indiretamente, a qualidade de vida de todo ser humano (DANTAS; ANDRADE, 2008; DUARTE; MORIHISA, 2008; FILIZOLA, 2006).

As pesquisas mostram um aumento do consumo do álcool por parte das mulheres. Esse dado também é corroborado em outros estudos desenvolvidos em nível local e internacional (CÉSAR, 2006; LARANJEIRA; DUALIBI; PINSKY, 2005; MIRANDA et al., 2007; NOVAES et al., 2000).

\section{Uso de álcool e mulheres}

A literatura tem apontado alguns fatores que podem estar envolvidos no aumento de consumo de álcool entre o gênero feminino, os quais parecem estar relacionados às mudanças no contexto da vida da mulher, seu papel social e ampliação de atividades e responsabiidades. Esse crescimento está associado às mudanças de comportamento social e à entrada da mulher no mercado de trabalho, na vida política (DANTAS; ANDRADE, 2008; MELONI; LARANJEIRA, 2004; OLIVEIRA; SANTOS, 2007).

A maior ocorrência (acima dos 16\%) de dependência do álcool foi encontrada nas regiões Norte e Nordeste. Fato mais preocupante foi a informação de que 5,2\% dos 
adolescentes (12 a 17 anos) eram dependentes do álcool. No Norte e Nordeste, essa porcentagem ficou próxima dos $9 \%$ (CARLINI et al., 2002).

Os estudos em nível local mostram que há uma predisposição para o uso e abuso do álcool e uma tendência ao alcoolismo feminino. As limitações físicas ou crônicas, a baixa autoestima, os temores e as dificuldades para estabelecer relações interpessoais, a falta de habilidade para resolver problemas, a baixa tolerância à frustração, ao fracasso e à dor, a insegurança, a timidez e a instabilidade emocional são fatores que podem levar as mulheres ao consumo do álcool. Muitas mulheres casadas começam a beber na meia idade, quando seu papel de mãe e esposa é desafiado pela partida de seus filhos, Síndrome do ninho vazio, dissolução do casamento, aposentadoria, entre outros fatores. Desde que a mulher, após seu movimento de emancipação, ampliou seu espaço social, aumentou sua participação na disputa pelo mercado de trabalho, suas responsabilidades (mãe, esposa, dona de casa, provedora e profissional, além de outras), ocorreu uma modificação nos tipos e na gravidade das doenças que incidem sobre o sexo feminino. Doenças como o infarto do miocárdio, acidente vascular cerebral e outras mais frequentes no sexo masculino estão também incidindo de forma mais direta sobre as mulheres. As mulheres estão, cada vez mais, usando drogas lícitas e ilícitas, como: cigarro, álcool, antidepressivos, hipnóticos, ansiolíticos, maconha e cocaína entre outras. Aspectos socioculturais também influenciariam de forma particular no padrão de consumo de álcool entre as mulheres. Estudos têm mostrado que as mulheres acima de 40 anos com um passado de violência física/sexual, familiar e doméstica estariam mais predispostas a um maior consumo de bebidas alcoólicas (DUARTE; MORIHISA, 2008, FILIZOLA, et al., 2009; FORNAZIER; SIQUEIRA, 2006; NOVAES et al., 2000).

Vários estudos mostram que as mulheres bebem como forma de se livrar dos sintomas associados a quadros de depressão. Além disso, tentam o suicídio quatro vezes mais frequentemente do que as abstêmias. O tratamento do alcoolismo em mulheres é mais complexo, uma vez que essas apresentam sintomas associados à doença como conflitos familiares, ansiedade, depressão e problemas socioeconômicos. No entanto, quando a mulher procura tratamento tem maior probabilidade de recuperação, na medida em que reconhece a gravidade do problema e procura formas de enfrentá-lo, mesmo considerando que há poucos serviços que ofereçam atenção específica para elas 
(NÓBREGA; OLIVEIRA, 2005; NOVAES et al., 2000; PASSINI JÚNIOR, 2005; SOUZA; LIMA; SANTOS, 2008).

O fenômeno do uso de álcool entre as mulheres preocupa por dois motivos: a) a mulher é mais vulnerável ao álcool e tem problemas mais cedo; b) a indústria de bebida tem investido em propagandas de álcool para as mulheres a exemplo do que ocorreu com o tabaco. Um estudo de coorte constatou um maior risco relativo para suicídio e acidentes fatais entre mulheres que consumiam acima de três doses diárias de bebidas alcoólicas (NOVAES et al., 2000; PONCE et al., 2008).

Alguns fatores como a constituição física e as experiências emocionais da mulher, em suas relações familiares e sociais, contribuem para deixá-la mais vulnerável ao alcoolismo. As influências culturais reforçam ou estabelecem os padrões familiares, a predileção pelo abuso do álcool como o meio de obter alívio da ansiedade e da depressão (que realmente ocorrem muito mais em mulheres que em homens). Experiências posteriores de privação do apoio emocional podem agir, às vezes, repetitivamente, como precipitantes de períodos de etilismo ou do retorno ao álcool, por parte daquelas que abandonaram o hábito. As usuárias de álcool também sofrem mais riscos de desenvolver doenças cardiovasculares, câncer da mama, osteoporose e distúrbios psiquiátricos, entre outros (MELONI; LARANJEIRAS, 2004; NOVAES et al., 2000).

A preocupação com o impacto do abuso e dependência de álcool entre as mulheres, com suas particularidades tem sido objeto de várias pesquisas. Dentre as principais observações realizadas, destaca-se o fato de que o início e o aumento do consumo de álcool, entre as mulheres estudadas, era mais tardio; elas também relatavam mais tentativas de suicídio, além de menor utilização concomitante de outras drogas ilícitas comparativamente aos homens (DANTAS; ANDRADE, 2008).

O aumento tardio no consumo de álcool também foi encontrado em trabalho de Wojnar et al.citado por Novaes(2000), avaliando dados retrospectivos de 1.179 pacientes poloneses (13,8\% mulheres). Este mesmo estudo apontou para uma maior prevalência, entre as mulheres, de transtornos de personalidade coexistentes, transtornos depressivos, transtornos de ansiedade, além de abuso de benzodiazepínicos e barbitúricos. Ainda em estudo internacional com pacientes internadas por alcoolismo apontaram para o abuso frequente de mais de uma substância psicoativa entre as 
mulheres, principalmente analgésica e tranquilizantes. Em decorrência do baixo percentual de pacientes do sexo feminino que acessam e aderem ao tratamento constata-se a necessidade de diferenciar a assistência ao público feminino devido às dificuldades encontradas em participar de um programa em que o predomínio é de indivíduos do sexo masculino (NÓBREGA; OLIVEIRA, 2005).

Embora o costume de beber entre as mulheres tenha aumentado à medida que a sociedade se tornou mais permissiva com esse comportamento, essa permissão não modificou a maneira estereotipada e preconceituosa com que a sociedade encara a embriaguez feminina. Visto como um comportamento não adequado do ser feminino, que é focalizado pelos atributos como docilidade, fragilidade, afetividade, representando, portanto, ainda um desafio para maiores avanços na área de tratamento (NOVAES et al., 2000; SOUZA; LIMA; SANTOS, 2008).

Quanto ao consumo de bebidas alcoólicas em quantidades potencialmente prejudiciais, o I Levantamento dos Padrões de Consumo de Álcool na População Brasileira indicou que $40 \%$ dos homens e $18 \%$ das mulheres consumiram cinco ou mais doses de bebidas alcoólicas numa única ocasião pelo menos uma vez no último ano. Este padrão, definido como uso pesado episódico do álcool, apresenta associação positiva com problemas de saúde física e mental, bem como acidentes e violência e um maior risco relativo para suicídio e acidentes fatais entre mulheres que consumiam acima de três doses diárias de bebidas alcoólicas (LARANJEIRA et al., 2007).

Dados recentes confirmam que, mesmo que o consumo de álcool seja realmente menor entre as mulheres, seu impacto pode ser maior do que entre os homens, avaliado por meio do relato de problemas associados ao álcool. O organismo feminino metaboliza o álcool de forma diferente da dos homens e, por isso, as mulheres sofrem mais rápido os efeitos nocivos da bebida. Enquanto os homens levam 15 anos para ter problemas no fígado, as mulheres levam cerca de apenas 5 . As mulheres com problemas com o álcool sofrem mais riscos de desenvolver doenças cardiovasculares, câncer da mama, osteoporose e distúrbios psiquiátricos, entre outros (CHALUB; TELLES, 2006; OLIVEIRA; SANTOS, 2007).

A pergunta que norteou o estudo foi: que complicações os autores têm identificado sobre as consequências do uso de álcool no organismo das mulheres? 
Dada a importância deste tema, o trabalho teve como objetivo realizar um levantamento da literatura científica brasileira, publicada na Base de dados Scielo na forma de artigos que referiam o uso de álcool pelas mulheres, identificando, na produção científica dos últimos dez anos (2000 a 2009), a relação entre o consumo de bebidas alcoólicas e os principais problemas a ele relacionados.

\section{Material e métodos}

O estudo se caracterizou como Pesquisa Bibliográfica Integrativa definida como aquela que sumariza as pesquisas realizadas sobre determinado assunto, construindo uma conclusão a partir de muitos estudos realizados separadamente, mas que investigam problemas idênticos ou similares. Apresenta um desenho metodológico de aproximações sucessivas, considerando que a flexibilidade na apreensão dos dados garante o movimento dialético no qual o objeto de estudo pode ser constantemente revisto (SOUZA; SILVA; CARVALHO, 2010). Enfim, significa realizar um movimento de observância das etapas, de leitura, de questionamentos e de interlocução crítica, o que exige vigilância epistemológica.

Para a realização da pesquisa, estabeleceu-se uma amostra de conveniência para a coleta do material bibliográfico no período de 2000 a 2009, totalizando 10 anos, cuja apresentação dos dados foi feita utilizando apenas números absolutos.

Foi consultada a Base de dados Scielo como fonte de informação e acessada no período de novembro de 2009 a julho de 2010. A escolha desta Base de dados se justifica pelo fato desta concentrar publicações de outras Bases, nomeadamente a Literatura Latino Americana de Ciências da Saúde (LILACS), voltada para estudos na área da saúde.

Como critérios para seleção dos artigos, estabeleceram-se uma amostra de conveniência que incluiu o tempo de publicação, 2000 a 2009, o idioma português e o artigo ter sido feito por pesquisadores do Brasil. Na leitura dos resumos identificaram-se os temas abordados pelos autores e definidas seis categorias que agruparam os estudos realizados no período, apresentadas a seguir.

1. Álcool e Gravidez incluiu os resumos dos estudos que abordavam o consumo de álcool na gravidez, riscos para o concepto do uso do álcool pela gestante durante a gravidez, puerpério e lactação. 
2. Álcool e Violência incluiu os resumos de estudos que abordaram o uso de álcool e manifestações de comportamentos violentos, tais como agressões a companheiros (as), filhos e outros familiares, brigas nos vários espaços (ruas, festas, escolas, local de trabalho, trânsito etc.) homicídios, tentativas de suicídios e suicídios entre e usuários de álcool.

3. Álcool e Comorbidades Psiquiátricas englobaram os resumos de estudos, relatando as coocorrências do uso de álcool com transtornos psíquicos, depressão e stress, entre outros.

4. Álcool e Doenças Crônico Degenerativas abrangeu os resumos das pesquisas que mencionaram a presença do uso do álcool por sujeitos portadores de enfermidades como Diabete, Hipertensão, câncer e outras que fazem parte desse grupo.

5. Álcool e Doenças Infecciosas e Infectocontagiosas é a categoria que incluiu os resumos dos estudos que mencionaram a presença do uso de álcool por sujeitos portadores de enfermidades infecciosas ou infectocontagiosas (Tuberculose, Dsts/Aids, Hepatite).

6. Álcool e Comportamentos de Risco agrupou os resumos dos estudos que versaram sobre beber e dirigir, acidentes de trânsito, beber no trabalho, relações sexuais desprotegidas, entre outros comportamentos que se referem como aqueles relacionados com os estilos de vida dos sujeitos.

\section{Resultados}

Foram encontrados 304 (trezentos e quatro) resumos no período estudado (2000 a 2009), cujos resultados podem ser visualizados na tabela 1.

Na tabela abaixo pode-se observar que o ano de 2008 foi o de maior número de produção de artigos sobre álcool que inclui as mulheres nos resultados dos estudos (72 artigos).

Tabela 1 - Distribuição dos Resumos segundo Ano de Publicação e Categorias. Natal, 2000-2009.

\begin{tabular}{lccccccccccc}
\hline \multicolumn{1}{c}{ Categorias/Ano } & $\mathbf{2 0 0 0}$ & $\mathbf{2 0 0 1}$ & $\mathbf{2 0 0 2}$ & $\mathbf{2 0 0 3}$ & $\mathbf{2 0 0 4}$ & $\mathbf{2 0 0 5}$ & $\mathbf{2 0 0 6}$ & $\mathbf{2 0 0 7}$ & $\mathbf{2 0 0 8}$ & $\mathbf{2 0 0 9}$ & Total \\
\hline Álcool e Gravidez & 1 & - & 1 & - & 2 & 11 & 3 & 8 & 11 & 3 & 40 \\
Álcool e Violência & & - & 1 & - & 2 & 14 & 2 & 3 & 15 & 15 & 62 \\
Álcool e Comorbidades & 5 & & 4 & & 7 & 3 & 4 & 3 & 11 & 9 & 46 \\
Psiquiátricas & & & & & & & & & & &
\end{tabular}




\begin{tabular}{|c|c|c|c|c|c|c|c|c|c|c|c|}
\hline $\begin{array}{l}\text { Álcool e Doenças Crônico } \\
\text { Degenerativas }\end{array}$ & 3 & - & - & 1 & 2 & 3 & 8 & 7 & 6 & 5 & 35 \\
\hline $\begin{array}{l}\text { Álcool e Doenças Infecto - } \\
\text { Contagiosas }\end{array}$ & 3 & 6 & - & - & 2 & 2 & - & 2 & 6 & 1 & 22 \\
\hline $\begin{array}{l}\text { Álcool e Comportamentos } \\
\text { de Risco }\end{array}$ & 7 & 1 & 10 & 2 & 16 & 10 & 7 & 12 & 23 & 14 & 99 \\
\hline Total & 19 & 7 & 16 & 3 & 31 & 43 & 24 & 35 & 72 & 47 & 304 \\
\hline
\end{tabular}

Fonte: Base Scielo (2000-2009).

Em relação às categorias temáticas, na mesma tabela constata-se, ainda, que o Álcool e o Comportamento de Risco foram as categorias predominantes em termos de número de estudos em todos os anos pesquisados (99 artigos), seguidos de Álcool e Violência (62 artigos) e Álcool e Comorbidades Psiquiátricas (46 artigos). Na sequência, com números próximos, Álcool e gravidez, Álcool e Doenças Crônicas e Degenerativas e, por último, Álcool e Doenças Infecto - Contagiosas.

\section{Discussão}

O alcoolismo na mulher é uma importante questão atual e as possíveis correlações com outros agravos à saúde vem gerando, cada vez mais, interesse científico, como possível fator etiológico, de risco e/ou comorbidade (CÉSAR, 2006; MELONI; LARANJEIRAS, 2004; ZALESK, 2006; OLIVEIRA; SANTOS, 2007).

A leitura dos resumos inseridos na categoria denominada "Álcool e Comportamentos de Risco" agruparam situações como o uso do álcool e dirigir ou o consumo dessa substância em ambiente de trabalho. Mostrou, ainda, que tais comportamentos antes atribuídos, predominantemente, aos homens, nesses últimos anos, tem relatado, também, a presença de mulheres usuárias (CÉSAR, 2006; CHALUB;TELLES, 2006).

Outros estudos referem, ainda, a condução de veículos motorizados após consumo abusivo de bebida alcoólica, sendo essa proporção maior em homens, mas também em mulheres. A prática de dirigir após consumo abusivo de bebida alcoólica apresentou maior frequência na faixa etária entre os 25 e 34 anos e com escolaridade acima de 11 anos de estudo (MOURA et al., 2009).

Os resumos da categoria "Álcool e gravidez" mostram a mulher como populaçãoalvo exclusiva, dada à especificidade do evento que não inclui o sexo masculino. A associação alcoolismo e problemas na gravidez podem ser encontrados em várias 
pesquisas, mostrando que evidências de um consumo maior de álcool pelas mulheres são fatores de risco para baixo peso do recém-nascido e Síndrome Alcoólica Fetal - SAF, além de outros. Não há, portanto, uma quantidade segura de bebida alcoólica que possa ser consumida durante a gravidez. Os efeitos do álcool sobre o feto são de extrema gravidade, podendo provocar seqüelas permanentes (CÉSAR, 2006; PASSINI JÚNIOR, 2005).

Os agrupados em "Alcool e Violência" também mostram a presença da mulher como uma das protagonistas de eventos violentos. Relações entre o uso de bebidas alcoólicas e comportamentos violentos foram encontrados em vários estudos, constatando-se, ainda, ocorrência de maus-tratos infantis perpretados por agressor usuário abusivo de álcool. Diversas pesquisas coincidem na afirmação de uma associação entre transtornos do uso de substâncias psicoativas e criminalidade. É possível constatar a alta proporção de atos violentos quando o álcool ou as drogas ilícitas estão presentes entre agressores, suas vítimas ou em ambos. A violência e o consumo de drogas no ambiente famíliar e no contexto sociocultural se constituem aspectos do cotidiano dos sujeitos que possivelmente induziram as mulheres a se tornarem dependentes e influenciaram o seu papel de mãe. O álcool age frequentemente como um desinibidor, facilitando a violência e aumentando a frequência nos episódios de agressões, reduzindo o controle dos impulsos e aumentando os pensamentos paranoicos. As estatísticas apontam que em cerca de 15 a 66\% de todos os homicídios e agressões o agressor, vítima, ou ambos tinham ingerido bebidas alcoólicas. Da mesma maneira, o consumo de álcool está presente em cerca de 13 a 50\% dos casos de estupro e atentados ao pudor (FILIZOLA, et al., 2009; PONCE et al., 2008; ZILBERMAN; BLUME, 2005).

No Brasil, dados do Cebrid mostram que $52 \%$ dos casos de violência doméstica estavam ligados ao álcool (CÉSAR, 2006; CHALUB;TELLES, 2006; LARANJEIRAS; DUALIBI; PINSKY, 2005).

O alcoolismo enquanto doença crônica traz consigo uma série de fatores de risco conforme apontado em um estudo que afirma que no Brasil $74,4 \%$ da população já fez uso de álcool alguma vez na vida (CARLINI et al., 2002). O consumo dessa substância tem sido associado ao comportamento sexual de risco para a transmissão da AIDS e outras DSTs (OLIVEIRA; SANTOS, 2007). 
Entretanto, cumpre assinalar que nas demais categorias há registro do aparecimento do sexo feminino em todos os resumos dos estudos agrupados, mostrando que há co-ocorrencia de outras doenças com o uso do álcool, também, entre as mulheres (DANTAS; ANDRADE, 2008, LUIS; LUNETA; FUZA, 2005).

A AIDS e a tuberculose foram frequentes, constituindo-se doenças associadas ao alcoolismo em mulheres, embora haja predomínio de homens (JOAQUIM; CARANDINA; DEFAVERI, 2006).

Há associação entre dependência de álcool e manifestação de transtornos mentais e psiquiátricos e de comportamento decorrentes do uso dessa droga. São encontrados como transtornos mais comuns: alimentares, de humor, da personalidade, depressão, tanto uni como bipolar, de ansiedade, de conduta, déficit de atenção e hiperatividade e a esquizofrenia, também apresentam estreita correlação com o abuso de substâncias, incluindo o álcool (LARANJEIRA; DUALIBI; PINSKY, 2005; PONCE et al., 2008; LUIS; LUNETTA; FERREIRA, 2008).

\section{Considerações finais}

As leituras dos resumos dos artigos produzidos, nos últimos dez anos, permitiram identificar várias categorias de estudos e mostraram que há necessidade de dispensar uma atenção especial, por parte dos gestores públicos, profissionais de saúde e familiares, às mulheres usuárias do álcool. Isto se justifica pela gravidade do problema que se caracteriza como de saúde pública, pelos aspectos emocionais, comprometimentos clínicos, psicológicos e emocionais. Esse conjunto de atenções pode possibilitar a promoção da autoestima e o resgate da cidadania, objetivando a melhor continuidade do processo de tratamento, recuperação e prevenção.

A crescente incidência e prevalência de consumo de substâncias psicoativas nas mulheres, inclusive nas jovens e adolescentes, e a alteração nos padrões de consumo constituem uma ameaça à sua saúde física, bem-estar e qualidade de vida. O alcoolismo, como quase todas as doenças, apresenta sinais e uma sintomatologia que abrange várias etapas, e quando não tratada pode ser fatal.

Concluí-se pela necessidade de mais estudos sobre a temática, incluindo o gênero feminino como uma importante variável a ser considerada nas políticas públicas de 
enfrentamento e combate bem como nas estratégias de prevenção e/ou tratamento. Embora cresça o número de mulheres com abuso ou dependência alcoólica, elas ainda permanecem como alvo não prioritário na tomada de decisão dos gestores de políticas públicas.

\section{Referências}

BRASIL. Prevenção ao uso de álcool e outras drogas no ambiente de trabalho: conhecer para ajudar. Brasília: SENAD/SESI, 2008.

CARLINI, E. A.; GALDURÓZ, J. C. F. (Coord.). II Levantamento domiciliar sobre uso de drogas psicotrópicas no Brasil: estudo envolvendo as 108 maiores cidades do país - 2005. Brasília: Secretaria Nacional Antidrogas, 2007.

CARLINI, E. A et al. I Levantamento domiciliar sobre o uso de drogas psicotrópicas no Brasil: estudo envolvendo as 107 maiores cidades do país. São Paulo: CEBRID, 2002.

CESAR, B. A. L. Alcoolismo feminino: um estudo de suas peculiaridades: resultados preliminares. Jornal Brasileiro de Psiquiatria, Rio de Janeiro, v. 55, n. 3, 2006.

CHALUB, M.; TELLES, L. B. Álcool, drogas e crime. Revista Brasileira de Psiquiatria, São Paulo, v. 28, out. 2006.

DANTAS, H. S.; ANDRADE, A. G. Comorbidade entre transtorno de estresse póstraumático e abuso e dependência de álcool e drogas: uma revisão da literatura. Revista de Psiquiatria Clínica, São Paulo, v. 35, 2008.

DUARTE, C. E.; MORIHISA, R. S. Experimentação, uso, abuso e dependência de drogas. In: Prevenção ao uso de álcool e outras drogas no ambiente de trabalho: conhecer para ajudar. Brasília: SENAD/SESI, 2008. p. 41-49.

FILIZOLA, C. L. A. et al. Alcoolismo e família: a vivência de mulheres participantes do grupo de autoajuda Al-Anon. Jornal Brasileiro de Psiquiatria, Rio de Janeiro, v. 58,n. 3, 2009.

FILIZOLA, C. L. A. et al. Compreendendo o alcoolismo na família. Escola Anna Nery Revista de Enfermagem, Rio de Janeiro, v. 10, n. 4, p. 660-670, 2006.

FORNAZIER, M. L.; SIQUEIRA, M. M. Consulta de enfermagem a pacientes alcoolistas em um programa de assistência ao alcoolismo. Jornal Brasileiro de Psiquiatria, Rio de Janeiro, v. 55, n. 4, 2006.

GUIMARÃES, V. V. et al. Consumo abusivo e dependência de álcool em população adulta no estado de São Paulo, Brasil. Revista Brasileira de Epidemiologia, São Paulo, v. 13, n. 2, p. 314-325, 2010. 
JOAQUIM, A. F.; CARANDINA, L.; DEFAVERI, J. Tuberculose em necropsias realizadas no Serviço de Anatomia Patológica da Faculdade de Medicina de Botucatu. Jornal Brasileiro de Patologia e Medicina Laboratorial, Rio de Janeiro, v. 42, n. 3, jun. 2006.

LARANJEIRA, R.; DUAILIBI, S. M.; PINSKY, I. Álcool e violência: a psiquiatria e a saúde pública. Revista Brasileira de Psiquiatria, São Paulo, v. 27, n. 3, set. 2005

LARANJEIRA, R. et al. (Coord.). I Levantamento Nacional sobre os padrões de Consumo de Álcool na população Brasileira. Brasília: Centro Brasileiro de Informações Sobre Drogas Psicotrópicas, 2007.

LUIS, M. A. V.; LUNETTA, A. C. F.; FERREIRA, P. S. Protocolo para avaliação da síndrome de abstinência alcoólica por profissionais de enfermagem nos serviços de urgência: teste piloto. Acta Paulista de Enfermagem, São Paulo, v. 1, n. 1, p. 39-45, 2008.

LUIS, M. A. V.; LUNETTA, A. C. F.; FUZA, A. C. Álcool e outras drogas: levantamento preliminar sobre a pesquisa produzida no Brasil pela enfermagem. Revista Latino-Americana de Enfermagem, Ribeirão Preto, n. 13, p. 1229-1230, nov./dez. 2005.

MELONI, J. N.; LARANJEIRA, R. Custo social e de saúde do consumo do álcool. Revista Brasileira de Psiquiatria, São Paulo, v. 26, maio. 2004.

MIRANDA, F. A. N. et al. Predisposição ao uso e abuso e álcool entre estudantes de graduação em enfermagem da UFRN. Escola Anna Nery Revista de Enfermagem, Rio de Janeiro, v. 11, n. 4, dez. 2007.

MOURA, E. C. et al. Direção de veículos motorizados após consumo abusivo de bebidas alcoólicas, Brasil, 2006 a 2009. Revista de Saúde Pública, São Paulo, v. 43, n. 5, out. 2009.

NÓBREGA, M. P. S. S.; OLIVEIRA, E. M. Mulheres usuárias de álcool: análise qualitativa. Revista de Saúde Pública, São Paulo, v. 39, n. 5, out. 2005.

NOVAES, C. et al. Impacto do alcoolismo em mulheres: repercussões clínicas. Revista de Psiquiatria Clínica, São Paulo, v. 27, n. 1, jan./fev. 2000.

OLIVEIRA, J. F. P.; SANTOS, M. Vulnerabilidade de mulheres usuárias de drogas ao HIV/AIDS em uma perspectiva de gênero. Escola Anna Nery Revista de Enfermagem, Rio de Janeiro, v. 11, n. 4, dez. 2007.

PASSINI JÚNIOR, R. Consumo de álcool durante a gestação. Revista Brasileira de Ginecologia e Obstetrícia, Rio de Janeiro, v. 27, n. 7, jul. 2005.

PONCE, J. C. et al. Álcool em vítimas de suicídio em São Paulo. Revista de Psiquiatria Clínica, São Paulo, v. 35, 2008.

SOUZA, J. G.; LIMA, J. M. B.; SANTOS, R. S. Alcoolismo feminino: subsídios para a prática profissional da enfermagem. Escola Anna Nery Revista de Enfermagem, Rio de Janeiro, $v$. 12, n. 4, p. 622-629, dez. 2008. 
SOUZA, M. T.; SILVA, M. D.; CARVALHO, R. Revisão integrativa: o que é e como fazer. Einstein, São Paulo, v. 8, n. 1, p. 102-106, 2010.

ZALESK, M. Diretrizes da associação brasileira de estudos do álcool e outras drogas (ABEAD) para o diagnóstico e tratamento de comorbidades psiquiátricas e dependência de álcool e outras substâncias. Revista Brasileira de Psiquiatria, São Paulo, v. 28, n. 2, jun. 2006.

ZILBERMAN, M. L.; BLUME, S. B. Violência doméstica, abuso de álcool e substâncias psicoativas. Revista Brasileira de Psiquiatria, São Paulo, v. 27, out. 2005.

Recebido em: 18/04/2012

Aprovado em: 11/06/2012 